\title{
ANALYSIS STUDY ON CACHING AND REPLICA PLACEMENT ALGORITHM FOR CONTENT DISTRIBUTION IN DISTRIBUTED COMPUTING NETWORKS
}

\author{
Dr. Anna Saro Vijendran ${ }^{1}$ and S.Thavamani ${ }^{2}$ \\ ${ }^{1}$ Director of M.C.A, S.N.R SONS College, Coimbatore, Tamil Nadu, India. \\ sarovijierediffmail.com \\ ${ }^{2}$ Assistant Professor in Computer Applications, S.N.R SONS College, Coimbatore, Tamil \\ Nadu, India. \\ thavamaniphd11@gmail.com
}

\begin{abstract}
Recently there has been significant research focus on distributed computing network massively caching and replica placement problems for content distribution in globally. Peer-to-Peer $(P 2 P)$ network provides dynamically decentralized, self organized, scalable objects in distributed computing system. However such networks suffer from high latency, network traffic and cache update problems. The existing caching and replica placement techniques for placing objects across peer-to-peer network have no complete solution to these problems. This paper presents an overview of the current challenges present in P2P overlay networks, followed by describes briefly the analysis study of the existing algorithms and their merits and demerits. And also suggest a new popularity based QoS-aware(Quality of Service) smart replica placement algorithm for content distribution in peer-to-peer overlay networks which overcomes the access latency, fault tolerance, network traffic and redundancy problems with low cost. The new algorithm suggested is based on the outcome of the analysis study.
\end{abstract}

\section{KEYWORDS}

Distributed computing network, Peer-to-Peer network, Replica placement, Decentralized, Access Latency, and Network traffic, Redundancy, Fault tolerance.

\section{INTRODUCTION}

A distributed computing system consists of multiple software components that are on multiple computers, but run as a single system [12, 19]. Internet is uprising as a new platform for distributed computing. Each computer in a distributed system is connected by a local network, or by a wide area network; interact with each other in order to achieve a common goal. A distributed system consists of any different possible configurations [19].

In recent years, the emergence of Internet-scale distributed systems including storage administration in global companies, entertainment file sharing, and large distributed database systems have led to extensive research on efficient and scalable distributed computing architectures. Among the other distributed computing models, P2P computing exhibits good scalability and stability [4]. P2P system is an alternative to conventional client/server systems and it supports applications that offer file sharing and content exchange. A node (peer) may act as a 
client or a server or router when requested or served or forwarded an object. The participating peers mark at least part of their resources as 'shared', allowing other contributing peers to access these shared resources. As a result, as new peers access a particular file, the system's capacity to provide that file increases [12]. P2P system proved to be an efficient and successful way for content distribution in distributed computing and file sharing over the Internet [4].

P2P systems are classified into two types namely; Centralized P2P system and Decentralized P2P system. The paper mainly deals with decentralized P2P system. Decentralized P2P system can be classified into decentralized structured and unstructured system. In Decentralized Structured P2P architecture network topology is tightly controlled, whereas Decentralized Unstructured P2P systems do not have any control over the network topology, and placement files over the network. The most general P2P system is the decentralized unstructured system [18]. Peers form a network among them on top of the existing inter infrastructure, which is known as the Overlay Network [6,22]. The challenges facing in P2P systems are scalability, reliability, access latency, network traffic, fault tolerance, bandwidth utilization, security, and load balancing.

Data replication and caching techniques are the important two services in distributed computing networks. It increases data availability by creating local or nearly available copies of popularly used items, by forwarding each query to its nearest copy; the query search latency can be effectively reduced. It also reduces communication overhead, increased system performance, achieves fault-tolerance, and enhances reliability and load balancing [10].

The web content and streaming media is the growing importance in efficient distribution. To provide content distribution to the clients with good Quality of Service (QOS), retaining efficient and balanced resources is a very big challenge [21]. QOS is a method to guarantee a bandwidth relationship between individual applications or protocols. It minimizes access latency, cost and increase the availability through replication technique. There are two classes of service models namely: Replica-Aware Service (RAS) model and Replica-Blind Service (RBS) model. In RAS model, the servers in the system are aware of the locations of replicas. By using this information's the servers direct the request to the nearest replica of the target object. In RBS model, the servers in the system are not aware of the locations of replicas or even their existence. So, request routing is independent of where the replicas of the target object are placed. Each replica only serves the requests flowing through some routing techniques which can be implemented at either the application level or at the network level [20].

\section{REPLICATION}

Replication is the process of creation and maintenance of duplicate copies of objects in internetscale distributed system. Replication improves the system performance, fault tolerance, reduces a network bandwidth usage and increases the availability of popular data objects by distributing the source of information in globally [13]. To efficiently use the server storage we need to replicate objects that will yield the best performance [17]. Replication is needed in the case of System failure, network traffic and to increase system scalability, load balancing, and to reduce access cost. For example, users can access a local object rather than origin server to minimize network traffic, access latency and provide location transparency [13].

There are different models of object replication. We mainly deal with a distributed replication group. A distributed replication group contains several servers dedicating some storage for the replicas. A server has to serve requests from its clients and also from other servers in the group. When a server receives a request from a client, it immediately responds to the client if the object is in its local storage. Otherwise, the object is fetched from other servers within the group at a higher access cost or from the origin server, at an even higher cost; in the case no server within 
the group stores a replica of the object [2]. The purpose of the replication group is to achieve minimum access cost $[8,17]$. Among different methods of object replication, we have analyzed only some methods of replica placement problems. And also pros and cons of the replication techniques are analyzed and summarized in Table 1.

\section{RESEARCH ISSUES IN REPLICATION TECHNIQUES}

In this paper we have analyzed only some methods of replica placement problems in decentralized unstructured P2P systems are as follows:

\subsection{Replication Algorithms in a Remote Caching Architecture (RARCA) [1]}

This paper talks about distributed object replication policies that are designed to implement different optimization goals and the availability of high speed interconnections for the potential of resource sharing. Resource sharing takes place in the remote sites. The ability to access the objects cached at the remote sites are done at the remote memory. A Remote Caching Architecture (RCA) uses a remote memory by allowing all sites in the system to take the advantages of each other's local memory. RCA focuses on what objects should be cached at what site instead of simply caching objects on a demand basis. In RCA's remote memory are not mapped into a single coherent virtual memory space.

\subsection{QoS-Aware Intelligent Replica Management Architecture for Content Distribution in P2P Overlay Networks (QIRMA) [2]}

In this paper the author deals with Intelligent Replica placement algorithm, which the requested contents are classified into class I and class II. In class I most frequently accessed contents are replicated in strong cluster which is having high weight values and in number copies. In class II least frequently accessed contents are replicated in weak cluster which is having low weight values and in less number of copies. Routing is performed hierarchically by broadcasting the query only to the strong clusters. This method has a caching technique, to reduce the search latency. Using this method System scalability can be improved by distributing the load across multiple servers which is proposed by replication. The demanded contents can be brought much closer to the clients through replication techniques, thus reducing both the access latency and network traffic and increase the system performance by avoiding hot peers which becomes a bottleneck. The limitation in this method is that in strong clusters a few replicated items have more number of copies. So, this consumes more cost, memory space, bandwidth and redundancy. In weak clusters a few replicated items have less number of copies. Increased user response time and performance are the bottleneck of the system.

\subsection{Clustered KCenter: Effective Replica Placement in P2P Systems (CKERP) [4]}

In this paper the authors have proved that the replica placement problem in P2P networks has represented as a Clustered K-Center problem and is proven to be NP-complete. To overcome this problem, the author developed an approximation algorithm in the form of a distance graph for the network topology; the replica placement solution has built out of $(\mathrm{m}-1)$ power of current distance graph. This system also considers a group of nodes but not the individual system.

\subsection{Replica Placement Algorithm in Content Distribution Network (RPACDN) [5]}

This paper deals with replica placement problems in CDN. The author models the CDN consisting of CDN server's, which can store replicas and deliver replicas to other CDN server's 
whenever necessary. The users of the CDN's are considering in a clustering view in order to minimize the total cost of the network. The algorithm used by the author includes three parts namely replication algorithm preprocess, constraint $\mathrm{p}$-median model and algorithm of solving constraint p-median problems with the iteration method. The results specify that the CPM algorithms perform better than Random algorithm with less total cost.

\subsection{Distributed Selfish Replication (DSR) [8]}

In this work their main contribution is deriving Equilibrium Object (EO) placement strategies that improves local utilities for all nodes concurrently than local utilities under greedy local object placement, do not suffer from object mistreatment problems, and provides only demanded content instead of complete information to all nodes by applying EO strategies. They described a TwoStep Local Search (TSLS (k)) algorithm which employees Bloom Filters to distribute object, selected for replication in demanded patterns. TSLS $(\mathrm{k})$ works in a round- robin fashion that allows each node to perform up to $\mathrm{k}$ rounds to improve the current placement of objects till it receives the final copy of demanded object. It reduces bandwidth, access cost. It does not consider storage capacity on the node, number of rounds needed to replicate the complete demanded object which consumes more time to replicate.

\subsection{Distributed Selfish Caching (DSC) [9]}

In Distributed selfish caching deals with loosely coupled group of nodes to share their resources in order to achieve higher efficiency and scalability. Here the author talks about two causes of mistreatments namely, mistreatment due to cache state interactions between various members of the group and mistreatment due to the use of common scheme for cache management across all members of the group. The author suggest that on demand distributed caching is fairly resilient to the onset of mistreatment as long as proxying is not enabled and intra-group access cost do not include outliers. .

\subsection{Fast Replication in Content Distribution Overlays (FRCDO) [3]}

SPIDER (Spatial Indirection for path Diversity for Expedited Replication) employs two orthogonal components to minimize the maximum time to replicate to all destination sites by creation of multiple dynamic distribution trees using Transit Nodes. End to end reliable data transport. In this algorithm content is not directly pushed to the clients but replicated only to the small set of edge servers, catches or data centers. Through edge servers client fetches the data. Thus it reduces the make-span in the data replication.

Make-span $($ Time) $=$ the moment the data download completes at the last destination - The moment that the data transfer is initiated.

Here trees can be reconfigured quickly without losses. It overcomes Transit Node failures. Since tree construction by this system does not consider the bandwidth of different paths it leads to low quality trees. It also does not consider the amount of data transfer which may leads to tree bottleneck.

\subsection{Distributed Algorithm for Web Content Replication (DAWCR) [16]}

This method deals with caching and replication of most popular web content which reduces bandwidth usage and the access latency by designing a distributed approximation algorithm. The main objective of this algorithm is to improve the efficiency of popular web content replication within a distributed replication group. The group may consist of several servers that spent some 
memory space for replicated web content which is requested by their clients. When a server receives a request from its client, it response if the web content is in its local storage otherwise it fetches from other replication servers within the group or from the origin server. In this method the replicas are placed by considering the availability of server capacities and the request rates for web content. Replication of popular web content at a server much closer to the user could reduces the access time and network bandwidth. Thus it increases the overall system performance. By comparing with centralized algorithm this algorithm shows only one percentage degradation of system performance. And also does not provide the number of copies of replicated content.

\subsection{Distributed Algorithm for the Replica Placement Problem (DARPP) [17]}

Caching and Replication of most popular data objects results in reduction of network bandwidth usage and the access latency by designing a distributed approximation algorithm. The main focus is to improve the efficiency of popular object replication within a distributed replication group. The group may consist of several servers that allocate certain amount of memory to place the replicated object which is requested by their clients. When a server receives a request from its client, it response if the object is in its local storage otherwise it fetches from other replication servers within the group or from the origin server. By analyzing the request rates for objects and the server capacities the replicas are placed by considering the availability of server capacities. Replication of popular data objects at a server closer to the user reduces the access time and network bandwidth. Thus it increases the overall system performance with all objects. By comparing with centralized algorithm this shows only one percentage degradation of system performance and not decided how many copies of objects should be replicated in the group.

\subsection{On Replica Placement for QoS-Aware Content Distribution Method (RPQACD) [20]}

In this method several algorithms like greedy algorithm, tree based algorithm have been proposed for solving the web server replica placement problem. This method optimizes an average access latency of all clients that were in the online Content Distribution Network (CDN). Growing importance needs good Quality of Service (QOS) while retaining efficient and balanced resource consumption of the underlying infrastructure. In this method they consider replica-aware service model for directing requests to the nearest replica of the target object with the help of the location information's. From the study of this method it is seen that the average performance measurement of the entire clients have been calculated and not the individual.

\subsection{Dynamic Replica Placement for Scalable Content Delivery (DRPSCD) [21]}

In this technique a balanced dissemination-tree (d-tree) is proposed which reduces the number of replicas deployed by comparing several replica placement algorithms. A dynamic web content distribution system (d-tree) built on top of a peer-to-peer overlay network called tapestry has need developed. This is used to find nearby replicas for the client. This novel algorithm meets the clients request by placing minimum replicas to the closest systems with the consideration of QOS and server capacity. A node in a d-tree maintains state root server. Thus, it reduces QOS matrices vise, access delay, bandwidth consumption and improve information only for its parent and client children. So, client gets content from its children without contacting the scalability. But all these are achieved with limited local network topology. 


\subsection{An Overlay Network for Replica Placement within a P2P VoD Network (ONRPP2P) [6]}

In this paper the author have proposed techniques and algorithms for point-to-point streaming in autonomous systems as it might occur in large companies, a campus or even in large hotels. Their major aim was to create a replica situation that inter-sub network RSVP streams are reduced to a minimum. They have introduced the architecture of an overlay network for interconnecting sub networks. Each sub network contains a Local Active Rendezvous Server (LARS), not only act as directory server but also controls availability of movie content in its subnet work. Due to this, they have considered data placement strategies depending on restrictions of network bandwidth, peer capabilities, and also movie's access frequency.

\subsection{On the Placement of Web Server Replicas (PWSR) [11]}

In this paper the authors have proposed that several algorithms like greedy algorithm, tree based algorithm for solving the web server replica placement problem that optimizing an average access latency of all clients that were in the online CDN's. From the analysis we came to know that the author calculated the average performance measurement of the entire clients but it does not provide the individual system performance.

\subsection{The Cache Location Problem (CLP) [7]}

In this paper the author talks about the problem of where to place net-work caches, these location problems both for general caches and for transparent en-route caches (TERCs), and identify that are intractable. They had given Greedy optimal algorithms for line and ring networks, and present closed form formulate for some special cases. And also they presented a model for a Network and computationally efficient dynamic programming algorithm for the single web server that wishes to minimize the average access delay. This Greedy algorithm greedily replaces some already assigned caches with caches to improve the objective cost function. They had observed that a small number of TERCs are sufficient to reduce the network traffic significantly and to reduce traffic load in their network. TERCs have the advantage that they do not require changes (like a proxy assignment) by a user, and are easy to install and manage locally within a provider network. The main problem in evaluating any multi-server placement algorithm is that it is harder to obtain general network web traffic data.

\subsection{Heuristic Replica Placement Algorithms in Content Distribution Networks (HRPACDN) [23]}

In this paper the author proposed a new optimization model with server storage capacity constraints for the replica placement problems. They divide the replica placement problems into two sub-problems: the number of each replica and which servers to store the replicas. And also they designed an efficient algorithm (CPM) for allocate replicas of files to minimize the total cost. It includes three parts: replication algorithm pre-process, constraint P-median model and algorithm of solving constraint P-median problems which were solved by iteration methods. This CPM algorithm performs better than random algorithm with less total cost. 
International Journal of Peer to Peer Networks (IJP2P) Vol.3, No 6, November 2012

\subsection{Tables}

Table 1. Pros and Cons of the various replication techniques are summarized below

\begin{tabular}{|c|c|c|c|c|c|c|}
\hline $\begin{array}{l}\text { Algori } \\
\text { thm }\end{array}$ & Author & Year & $\begin{array}{c}\text { Journa } \\
\text { ls }\end{array}$ & Advantages & $\begin{array}{c}\text { Improved } \\
\text { Parameters }\end{array}$ & Limitations \\
\hline $\begin{array}{l}\text { DARP } \\
\text { P [17] }\end{array}$ & $\begin{array}{l}\text { S.Zaman } \\
\& \\
\text { D.Grosu. }\end{array}$ & 2011 & IEEE & $\begin{array}{l}\text { Minimizes } \\
\text { Response Time, } \\
\text { Cost \& Increase } \\
\text { Availability }\end{array}$ & $\begin{array}{l}\text { Cost, access } \\
\text { latency, } \\
\text { Network } \\
\text { bandwidth }\end{array}$ & 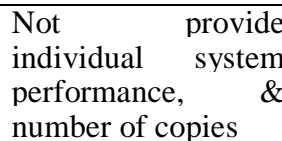 \\
\hline $\begin{array}{l}\text { HRPA } \\
\text { CDN[ } \\
23]\end{array}$ & $\begin{array}{l}\text { J.Sun, } \\
\text { S.Gao \& } \\
\text { at el. }\end{array}$ & 2011 & JNW & $\begin{array}{l}\text { Less total cost, } \\
\text { Better than random } \\
\text { algorithm }\end{array}$ & $\begin{array}{l}\text { Network } \\
\text { bandwidth, } \\
\text { Access cost }\end{array}$ & Limited Topology \\
\hline $\begin{array}{l}\text { RPAC } \\
\text { DN [5] }\end{array}$ & $\begin{array}{l}\text { J.Sun, \& } \\
\text { at el }\end{array}$ & 2010 & IEEC & $\begin{array}{l}\text { Minimize total } \\
\text { cost }\end{array}$ & Access cost & $\begin{array}{l}\text { Can't adapt dynamic } \\
\text { user request }\end{array}$ \\
\hline $\begin{array}{l}\text { QIRM } \\
\text { A [2] }\end{array}$ & $\begin{array}{l}\text { S.Ayyas } \\
\text { amy \& } \\
\text { S.Nataraj } \\
\text { an }\end{array}$ & 2009 & IJCSE & $\begin{array}{l}\text { Access latency, } \\
\text { Network traffic, } \\
\text { Scalability, Fault } \\
\text { Tolerance, Search } \\
\text { Latency }\end{array}$ & $\begin{array}{l}\text { Throughput, } \\
\text { Delay, } \\
\text { Bandwidth, } \\
\text { Query } \\
\text { Efficiency, }\end{array}$ & $\begin{array}{l}\text { High availability, } \\
\text { Redundancy, Low } \\
\text { Availability, } \\
\text { Bottleneck Problem, }\end{array}$ \\
\hline $\begin{array}{l}\text { DAW } \\
\text { CR } \\
{[16]}\end{array}$ & $\begin{array}{l}\text { S.Zaman } \\
\& \\
\text { D.Grosu. }\end{array}$ & 2009 & IEEE & $\begin{array}{l}\text { Response } \\
\text { Cost, Inerease } \\
\text { Availability } \quad \& \\
\text { Performance }\end{array}$ & $\begin{array}{l}\text { Access time, } \\
\text { Cost, } \\
\text { Network } \\
\text { Bandwidth }\end{array}$ & $\begin{array}{lr}\text { not } & \text { nrovide } \\
\text { individual } & \text { svstem } \\
\text { nerformance } & \& \\
\text { number of replicated } \\
\text { copies }\end{array}$ \\
\hline $\begin{array}{l}\text { DSC } \\
{[9]}\end{array}$ & $\begin{array}{l}\text { N.Laouta } \\
\text { ris, \& at } \\
\text { el. }\end{array}$ & 2007 & IEEE & $\begin{array}{l}\text { Improve scalability } \\
\text { and higher } \\
\text { efficiency }\end{array}$ & $\begin{array}{l}\text { Access } \\
\text { latency, } \\
\text { bandwidth } \\
\text { utilization }\end{array}$ & Vulnerability \\
\hline $\begin{array}{l}\text { CKER } \\
\text { PP2P } \\
{[4]}\end{array}$ & $\begin{array}{l}\text { J.Zhou } \\
\& \text { at el. }\end{array}$ & 2007 & IEEE & $\begin{array}{l}\text { Query Latency, } \\
\text { Faster than the } \\
\text { optimal solution }\end{array}$ & $\begin{array}{l}\text { Time } \\
\text { complexity }\end{array}$ & $\begin{array}{l}\text { Not consider the } \\
\text { individual system. }\end{array}$ \\
\hline $\begin{array}{l}\text { DSR } \\
{[8]}\end{array}$ & $\begin{array}{l}\text { N. } \\
\text { Laoutari } \\
\text { s \& at el. }\end{array}$ & 2006 & IEEE & $\begin{array}{l}\text { Replicate only } \\
\text { demanded pattern of } \\
\text { content, Network } \\
\text { Traffic, Access Cost }\end{array}$ & $\begin{array}{l}\text { Access } \\
\text { latency, } \\
\text { Bandwidth, } \\
\text { Delay }\end{array}$ & $\begin{array}{l}\text { Does not consider } \\
\text { storage canacitv. ton } \\
\text { manv rounds } \\
\text { additional nrotocols } \\
\& \text { mechanisms. }\end{array}$ \\
\hline $\begin{array}{l}\text { FRCD } \\
\text { O [3] }\end{array}$ & $\begin{array}{l}\text { S.Gangul } \\
\mathrm{y}, \& \text { at } \\
\text { el. }\end{array}$ & 2005 & IEEE & $\begin{array}{l}\text { Renlication time, } \\
\text { Transit Nodes } \\
\text { failover requirement }\end{array}$ & $\begin{array}{l}\text { Transfer } \\
\text { time, Speed }\end{array}$ & $\begin{array}{l}\text { Tree } \\
\text { problem }\end{array}$ \\
\hline $\begin{array}{l}\text { ONRP } \\
\text { P2P } \\
{[6]}\end{array}$ & $\begin{array}{l}\text { K.H. } \\
\text { wan \& at } \\
\text { el. }\end{array}$ & 2005 & JHPCN & $\begin{array}{l}\text { Access Freanencv, } \\
\text { reduce the number } \\
\text { of RSVP hased } \\
\text { inter-subnetwork }\end{array}$ & $\begin{array}{l}\text { Waiting } \\
\text { time }\end{array}$ & $\begin{array}{l}\text { Restrictions }{ }^{\mathrm{Nn}} \\
\text { network, expensive }\end{array}$ \\
\hline $\begin{array}{l}\text { ORPQ } \\
\text { ACD } \\
\text { M } \\
{[20]}\end{array}$ & $\begin{array}{l}\text { X.Tang } \\
\text { \& at el. }\end{array}$ & 2004 & IEEE & $\begin{array}{l}\text { Response time, } \\
\text { cost \& increase the } \\
\text { availability }\end{array}$ & $\begin{array}{l}\text { Throughput, } \\
\text { Latency, } \\
\text { Bandwidth, } \\
\text { Delay }\end{array}$ & $\begin{array}{l}\text { Does not provide } \\
\text { individual } \\
\text { performance } \\
\text { measurement }\end{array}$ \\
\hline $\begin{array}{l}\text { DRPS } \\
\text { CD } \\
{[21]}\end{array}$ & $\begin{array}{l}\text { Yan } \\
\text { Chen \& } \\
\text { at el. }\end{array}$ & 2002 & $\begin{array}{l}\text { Notes } \\
\text { in CS }\end{array}$ & $\begin{array}{l}\text { Reduces network } \\
\text { Traffic. Improve } \\
\text { Scalability }\end{array}$ & $\begin{array}{l}\text { Bandwidth } \\
\text { access delay }\end{array}$ & $\begin{array}{lr}\text { Limited local } \\
\text { network topology } \\
\text { knowledge }\end{array}$ \\
\hline $\begin{array}{l}\text { PWSR } \\
{[11]}\end{array}$ & $\begin{array}{l}\text { L.Qiu. } \\
\& \text { at el. }\end{array}$ & 2001 & IEEE & $\begin{array}{l}\text { Better performance, } \\
\text { Availability, } \\
\text { Request Latency, } \\
\text { Load Balancing }\end{array}$ & $\begin{array}{l}\text { Request } \\
\text { rate, } \\
\text { Bandwidth, } \\
\text { access delay }\end{array}$ & $\begin{array}{l}\text { Depends upon } \\
\text { estimates of Clients } \\
\text { Distance \& Load } \\
\text { Predictions }\end{array}$ \\
\hline $\begin{array}{l}\text { CLP } \\
{[7]}\end{array}$ & $\begin{array}{l}\text { P.Krishn } \\
\text { an, \& at } \\
\text { el. }\end{array}$ & 2000 & $\begin{array}{l}\text { IEEE/A } \\
\mathrm{CM}\end{array}$ & $\begin{array}{l}\text { Minimize overflow, } \\
\text { Do not require } \\
\text { changes, Easy to } \\
\text { install \& manage } 1\end{array}$ & $\begin{array}{l}\text { Average } \\
\text { Delay, } \\
\text { Network } \\
\text { Traffic } \\
\end{array}$ & $\begin{array}{l}\text { Limited Network } \\
\text { Topology, Does not } \\
\text { support worst case } \\
\text { level }\end{array}$ \\
\hline $\begin{array}{l}\text { RARC } \\
\text { A } \\
{[1]}\end{array}$ & $\begin{array}{l}\text { Avraham } \\
\text { Leff \& at } \\
\text { el. }\end{array}$ & 1993 & IEEE & $\begin{array}{l}\text { Reduces a number } \\
\text { of disk access, } \\
\text { improves } \\
\text { performance }\end{array}$ & $\begin{array}{l}\text { Throughput, } \\
\text { Latency, } \\
\text { Bandwidth, } \\
\text { Delay }\end{array}$ & $\begin{array}{l}\text { Deals with only } \\
\text { limited network } \\
\text { topologies }\end{array}$ \\
\hline
\end{tabular}




\section{SUgGESTION}

In order to address the limitations popularity based QoS-aware smart replica placement algorithm for content distribution in P2P overlay network is being developed based on weight vector values of peers and the accessing popularity of requested objects. The suggested algorithm contains a smart replica placement algorithm and modified robust query searching technique for data retrieval. The suggested system solves replica placement problem and is defined as follows: In this algorithm, according to the peer's weight vector values the peers are grouped as strong cluster, medium cluster and weak clusters. And followed by the requested objects are classified into Class I, Class II and class III. In class I, most frequently accessed objects are replicated in strong clusters which are having high weight values. In class II, medium accessed objects are placed in medium clusters which are having medium weight values. In class III, least frequently accessed objects are replicated in weak clusters and having low weight values. Routing is performed hierarchically by broadcasting the query to the strong, medium group of peers. In addition this algorithm also possesses caching techniques to the reduction of search latency. It minimizes the access time and reduces the memory space, cost, redundancy, bandwidth consumption. And also it increases the system performance and availability.

\section{CONCLUSIONS}

This paper conducts a theoretical analysis study on caching and replication strategies in distributed computing system (peer-to- peer network). A brief discussion of those techniques is summarized. The advantages and limitations of caching and replication techniques are summarized with reference to various issues related to caching and replication techniques in distributed computing networks.

\section{ACKNOWLEDGEMENTS}

Our thanks to AIRCC for allowing us to modify templates they had developed. And also our sincere thanks to our Management of SNR Sons Institutions for allowing us to utilize their resources for doing our research work. Our sincere thanks to our Principal \& Secretary Dr.H.Balakrishnan M.Com., M.Phil., Ph.D., for his support and encouragement in our research work. And my grateful thanks to my guide Dr. Anna Saro Vijendran, M.C.A., M.Phil., Ph.D., Director of M.C.A, SNR Sons College, Coimbatore-6 for her valuable guidance and giving me a lot of suggestions \& proper solutions for critical situations in our research work.

\section{REFERENCES}

[1]Avraham Leff, Joel L. Wolf, and Philip S. Yu, (1993) "Replication Algorithms in a Remote Caching Architectures", IEEE Trans. On Parallel and Distributed Systems, Vol.4, No.11, PP 1185-1204.

[2]S. Ayyasamy and S.N. Sivanandam,(2009) “ A QOS-Aware Intelligent Replica Management Architecture for Content Distribution in Peer-to-Peer Overlay Networks “, International Journal on Computer Science and Engineering, Vol.1, No.2, PP 71-77.

[3]S. Ganguly, A. Saxena, S. Bhatnagar, and R. Izmailov are with the Broadband and Mobile Networking Department,NEC laboratories, Princeton, NJ, 08540, USA,(2005) "Fast Replication in Content Distribution Overlays", SUBMITTED TO IEEE INFOCOM 2005.

[4]Jian Zhou, Xin Zhang, Laxmi Bhuyan and Bin Liu. (2007) "Clustered KCenter: Effective Replica Placement in Peer-to-Peer Systems", IEEE conference on Global Telecommunications, PP.20082013.

[5]Jing Sun, Suixiang Gao, Wenguo Yang, Fei Huang. (2010)," Replica Placement Algorithms in Content Distribution Networks", Information Engineering and Electronic Commerce (IEEC) 2nd International symposium on 23-25 July 2010. 
[6]Kan Hung Wan, Chris Loeser,(2005), "An Overlay Network for Replica Placement within a P2P VoD Network", Journal Of High Performance Computing And Networking, Vol.3, No.5/6, pp.320335,December 2005.

[7]P. Krishnan, D. Raz, and Y. Shavitt,(2000), “The cache location problem,” IEEE/ACM Transactions on Networking, vol. 8, no. 5, pp. 568-582, Oct. 2000.

[8]N. Laoutaris, O. Telelis, V. Zissimopoulos, and I. Stavrakakis.(2006). "Distributed Selfish Replication”, IEEE Transaction on Parallel and Distributed Systems. vol. 17, no. 12. PP.1401-1413 Dec. 2006.

[9]N. Laoutaris, G. Smaragdakis, A. Bestavros, and I. Matta. (2007).” Distributed Selfish Caching”, IEEE Trans. Parallel and Distributed Systems, Vol. 4, No.11,PP.1185-1204, Nov- 1993.

[10]Pooja Sharma, Kamal kant and Naveen Chauhan. July-Dec 2010. "A Comparative Study of ClusterBased Data Replication Techniques for MANETs “, International journal of information technology and knowledge management. Vol. 2, No. 2. (July-Dec 2010). 665-667.

[11]L. Qiu. , V. N Padmanabhan, and G. M., Apr. 2001 Voelker, "On the placement of web server replicas," in Proceedings of IEEE INFOCOM'01, pp. 1587-1596.

[12]Sabu M.Thampi. 2010. Survey of search and replication schemes in unstructured p2p networks. Network protocols and Algorithms. Vol.2, No.1. (2010).

[13]Salman Abdual Moiz at el. Jan-2011. "Database Replication: A Survey of Open Source and Commercial Tools", International Journal of Computer Applications. Vol. 13, No .6. (Jan-2011).

[14]Samee Ullah Khan and Cemal Ardil. 2009. "A Fast Replica Placement Methodology for Large-scale Distributed Computing Systems”, World Academy of Science, Engineering and Technology. 55. (2009).

[15]Saurabh Tewari. 2007. "Performance Study of Peer-to-Peer File Sharing" . Doctorial Thesis, University of California, Los Angeles. (2007).

[16]Sharrukh Zaman and Daniel Grosu. 2009." A Distributed Algorithm for Web content Replication”, 8th IEEE International symposium on network computing and applications. (2009).

[17]Sharrukh Zaman and Daniel Grosu. Sep-2011. "A Distributed Algorithm for the Replica Placement Problem", IEEE Trans.Parallel and Distributed Systems. vol. 22, no.9. ( sep-2011) 1455-1468

[18]Stephanos, Routsellis-Theotokis and Diomidis Spinellis. Dec-2004.” A Survey of Peer-to-Peer Content Distribution Technologies", ACM Computing Surveys. Vol. 36, No.4. (2004). 335-371.

[19]What is distributed computing? http://www.wikipedia.com

[20]Xueyan Tang Jianliang Xu. Mar-2004. “On Replica Placement for QoS-Aware Content Distribution”, INFOCOM 2004. 23rd AnnualJoint Conference of the IEEE Computer and Communications Societies. (March 2004). 7-11.

[21]Yan Chen, Randy H. Katz and John D. Kubiatowicz. 2002. "Dynamic Replica Placement for Scalable Content Delivery”. Lecture Notes in Computer Science, Vol. 2429. (2002). 306 318.

[22]Guruprasad Khataniar and Diganta Goswami, Sep-2012. " SHP: A Hierarchical Protocal to Improve Performance of Peer-to-Peer Systems", International Journal of Peer to Peer Networks.(September 2012). Vol.3, No5.

[23]Jing Sun, S.Gao, W.Yang and Z.Jiang, March-2011. "Heuristic Replica Placement Algorithms in Content Distribution Networks”, Journal of Networks. Vol.6, No.3,pp.416-423. (March 2011).

\section{Authors}

Dr. Anna Saro Vijendran is the Director - MCA in SNR Sons College, Coimbatore, India. She has a teaching experience of 20 years in the field of Computer science. Her area of Specialization is Digital Image Processing and Artificial Neural Networks .She has presented more than 30 Papers in various Conferences and her research works have been published in International Journals. She is currently a Supervisor for research works of various Universities and also Reviewer for reputed Journals.

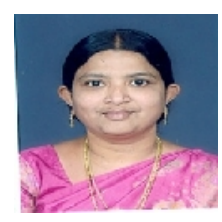

S, Thavamani is an Assistant Professor in Department of Computer Applications, SNR Sons College, Coimbatore, India. She has a teaching experience of 12 years in the field of Computer science. Her area of Specialization is Distributed Computing and Networks. She has presented more than 15 Papers in various International and National Conferences. She is currently a supervisor for M.Phil research works of various Universities. She is currently pursuing Ph.D Degree in SNR Sons College, under Bharathiar University, Coimbatore.

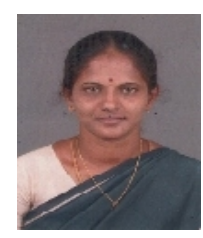

